\title{
Approach for Synthesis and Optimization of Complex Thermal Systems for Supermarkets
}

\author{
Jonathan Kistner ${ }^{1 *}$, Wilhelm Tegethoff ${ }^{1}$, Nicolas Fidorra² ${ }^{2}$, Jürgen Köhler ${ }^{2}$ \\ ${ }^{1}$ TLK-Thermo GmbH, Hans-Sommer-Straße 5, 38106 Braunschweig, Germany; *j.kistner@tlk-thermo.de \\ ${ }^{2}$ Institut für Thermodynamik, Technische Universität Braunschweig, Hans-Sommer-Straße 5, \\ 38106 Braunschweig, Germany
}

SNE 29(2), 2019, 93 - 100, DOI: 10.11128/sne.29.tn.10476

Received: April 15, 2019 (Selected ASIM GMMS/STS 2019

Postconf. Publ.), Accepted: June 10, 2019

SNE - Simulation Notes Europe, ARGESIM Publisher Vienna,

ISSN Print 2305-9974, Online 2306-0271, www.sne-journal.org

Abstract. The development of thermal systems for supermarkets is a challenging task. Both, heating and cooling demands at different temperature levels have to be satisfied under individual boundary conditions. In combination with a broad range of available technologies and components, a high number of possible system layouts exist. Thus, various types of refrigeration systems can be found in supermarkets: Central refrigeration systems with one or two stages and direct evaporation, central systems with a secondary loop or systems with (semi)-plug-in-cabinets. The system topology and operating strategy depend on climate conditions, building scale, customer's occupancy or evaluation criteria. In practice, established solutions based on experience are used. However, comparing all alternative concepts is difficult. Beside the consideration of investment costs, it is essential to evaluate the energy consumption. For the calculation of energy consumption, considering dynamic interactions between components is crucial.

To compare different system layouts under consideration of dynamic interactions, an optimal operating control has to be applied. Furthermore, the high number of possible topologies makes it necessary to reduce the complexity for the selection of components and their interconnections. Therefore, software based methods are needed to efficiently reduce complexity and evaluate system alternatives in a dynamic environment.

This paper presents a procedure that supports the user to find an optimal system topology under individual conditions. As an example, a secondary-loop refrigeration system with low and medium temperature cabinets is applied.
The user defines ambient conditions and requirements such as cooling load and temperature setpoints. Additionally, a set of transient, non-linear models for available technical equipment is defined. The parametrized, ready-to-use models are managed in a catalogue platform. In the catalogue, additional information is stored, like valid operational ranges, which is used during optimization. On this information basis, an algorithm deduces a reasonable refrigeration system layout. Intermediate result is a ready-to-simulate system. It contains only catalogue models that have physical reasonable interconnections. Subsequently, the system's fluid flow rate of each connection is optimized. The result of the optimization is used for evaluation of the system layout and further reduction of its topology.

The paper shows, that using simple input information, the complexity of the optimization problem can be extremely reduced. The suggested procedure is capable to deploy an optimal system topology under consideration of non-linear dependencies.

\section{Introduction}

Many engineering work is spent on developing better thermal energy systems. The effort that is being made touches all sectors of industry and science like cars with electrified drivetrains [14], busses [3][9] and supermarkets [1]. Especially for supermarkets, the energy saving potential is enormous. A broad range of different technologies is available. At the same time, many different thermal demands typically occur. In practice, established solutions based on experience are used. However, comparing all alternative concepts is difficult. The high number of possible topologies makes it necessary to reduce the complexity for the selection of components and their interconnections. Software based methods are needed to efficiently reduce complexity and evaluate system alternatives considering all relevant non-linearities. 
This paper presents an approach to support the user finding an optimal system topology under individual conditions. As an example, a secondary-loop refrigeration system with low temperature (LT) and medium temperature (MT) cabinets is applied. The system is synthesized on basis of ready parameterized catalogue models. A steady state parameter optimization of the synthesized system model is performed. Further system reductions are derived from the optimization result. The overall electrical energy consumption of system variants are statistically estimated over one year for different cities in Europe.

\section{Supermarket Refrigeration Systems}

Supermarket energy systems have big energy saving potentials. Energy savings related to the application of optimal topologies in combination with optimal operating strategies can be tentatively estimated to be in a magnitude of $20 \%$ [2][13].

Furthermore, energy systems of supermarkets typically possess a high complexity caused by many different requirements: The salesroom has to be cooled, heated and dehumidified. Groceries have to be kept at different low temperature levels. The optimal system topology changes with climatic boundary condition, building scale, customers' frequency and evaluation criteria. Improvements of energy efficiency and reduction of emissions are tried to achieve by applying waste heat recovery, regenerative technologies or thermal storages [12][8].

Beside detailed system variants, some general system types for supermarket refrigeration exist: Widely used and under intensive research are central refrigeration systems. Those systems can be huge refrigeration cycles with several evaporators at different temperature levels satisfying both cooling and freezing demands. The cabinets directly contain the evaporators. The main disadvantage is the high complexity of controls and a high charge of refrigerant. In water-loop refrigeration systems every cabinet contains its own little refrigeration cycle. The water loop transfers the waste heat of the condensers to the ambient. For low temperature cabinets, often a secondary brine loop with an additional refrigeration cycle is used to cool the cabinet's condenser. In secondary-loop systems, the cabinets are directly cooled by brine. Different refrigeration cycles provide brine at needed temperature setpoints. Water-loop and secondary-loop systems are more and more focused in current researches. Amongst others, the reasons are small amounts of refrigerant and relatively easy to control and combine in different layouts [1]. A comparison, especially with central refrigeration systems is of big interest in the current scientific and economic discussion [4].

The mentioned aspects encouraged the author to use a secondary-loop system as example for system synthesis and optimization. In fact, this is already a design specification. The example is chosen to be very simple to ease the evaluation of the represented procedure. This paper is a preliminary study for further methodical work to support scientists and planners to develop new system topologies for supermarkets.

\section{Procedure for Layout Development}

In this chapter, the applied procedure for layout development is represented. It contains three main topics: Firstly, set up of the information basis. Secondly, execution of a system synthesis based on the defined information. Thirdly, optimization of the synthesized system model.

Figure 1 shows the process of the layout development. In the first step, the user starts defining requirements and boundary conditions. At this point, the refrigeration loads and related temperature setpoints are defined. The boundary conditions can be connected to a specific location that is relevant for the system development. Generic boundary conditions are used in this paper. Furthermore, a set of components has to be chosen, that shall be available for the system synthesis. Most information related to the catalogued models can be assumed to be given as meta data. However, the user might have to change some data like temperature setpoints for refrigeration cycles or estimated valid inlet temperatures for cabinets. Once the system is synthesized, the user need to find valid starting conditions for optimization.

In the present study, three main questions were of particular interest:

- How could a synthesis look like on basis of component functionalities without executing any simulations?

- How does a well-suited information basis look like to efficiently support the system synthesis and reduce complexity?

- How can a system layout efficiently be evaluated with less user interactions as possible? 


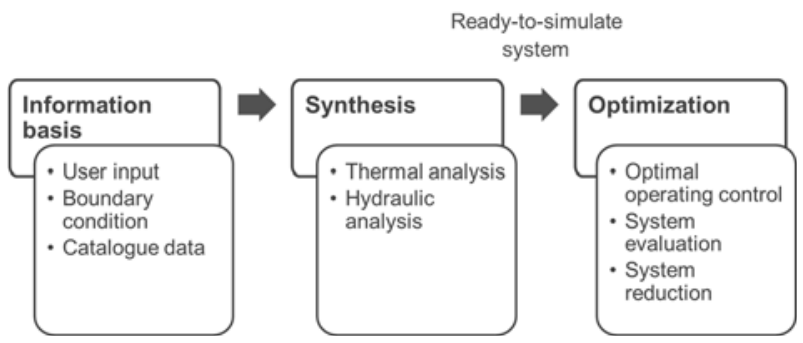

Figure 1: Employed procedure for system synthesis and optimization. The synthesis uses a well-suited information basis to find physical reasonable interconnections. The synthesized ready-tosimulate system model is optimized.

The used component models in this paper can be seen as type representatives. The procedure is not aimed to work for design tasks, which are typically characterized to have a big number of very similar components. Other procedures for system design exist, that could be applied consecutively or be integrated in the represented procedure in future work.

\section{Information Basis for System Synthesis}

As described in Chapter 2, the information basis contains requirements and specifications of the development task as well as a defined set of component models with related meta data. In this section, all basic information of the applied development task is specified and the available component models are described in detail.

\subsection{Catalogue models}

All available system components are modeled on basis of TIL [11]. Thus, the modelling language is MODELICA. TILMedia is used for media property calculation.

Figure 2 shows the component models. In the chosen scenario, the models for the cabinets are assumed to be part of the requirement defined by the user. The refrigeration units are inheritors from the same refrigeration cycle with modified parameters. The refrigeration cycle contains a physical based compressor model, two finite volume heat exchangers, an orifice valve and an ideal separator after the condenser. The whole cycle is scalable by one nominal cooling capacity. Additionally, it is capable to automatically switch on and off, depending on the boundary conditions at the condenser and evaporator. The compressor is controlled to maintain a fixed temperature setpoint at the brine outlet of the evaporator. The valve controls the superheat of the refrigerant at the evaporator outlet. The two refrigeration units mainly differ in their type of refrigerant, their scale and their temperature setpoint.

The outdoor units basically are modeled as simple temperature boundaries. The second outdoor unit additionally is capable of regulating the outlet temperature by mixing the ambient temperature with the inlet flow. The cabinets are modeled as heat boundary. An early design decision is made by using cabinet models only for secondary-loop systems.

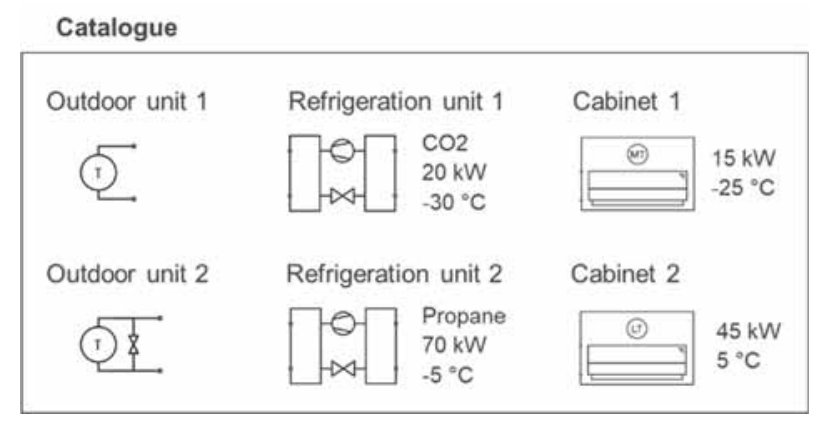

Figure 2: Catalogue models available for the system synthesis. The models for the cabinets are assumed to be part of the requirement. All other components are optional.

The catalogue models contain meta data that describe the functionalities and valid temperature ranges at all inand outlets. Detailed examples for the meta data used for system synthesis can be seen in Chapter 4 .

\subsection{Requirements and specifications}

The requirements and specifications are typically user inputs that characterize the overall optimization problem. As requirement, a refrigeration load with $45 \mathrm{~kW}$ at $5{ }^{\circ} \mathrm{C}$ and a load with $15 \mathrm{~kW}$ at $-25^{\circ} \mathrm{C}$ is specified. Furthermore, the user need to adjust the valid temperature ranges at the cabinets' inlet to complete the information basis for system synthesis. The temperature of the ambient air in a range of $-10{ }^{\circ} \mathrm{C}$ to $40{ }^{\circ} \mathrm{C}$ is defined as ambient condition. The supermarket building is not considered in this study.

\section{Synthesis of a Secondary-loop Refrigeration System}

The system synthesis represented in this paper uses the described information basis in Chapter 3 to deduce a physical reasonable system layout. In the first place, a thermal functional analysis for each inlet and outlet of the available components is executed.

$$
\text { SNE 29(2) - 6/2019 } 95
$$


Catalogued temperature information and thermal functionalities of each component are used to estimate reasonable interconnections. Secondly, a hydraulic analysis integrates additional components to complete the hydraulic network and make the model executable.

\subsection{Thermal analysis to find reasonable interconnections}

Figure 3 shows a simplified view on the meta data that is used for thermal system synthesis. Original catalogue data, defined setpoints and deduced requirements are combined as information basis for the system synthesis.

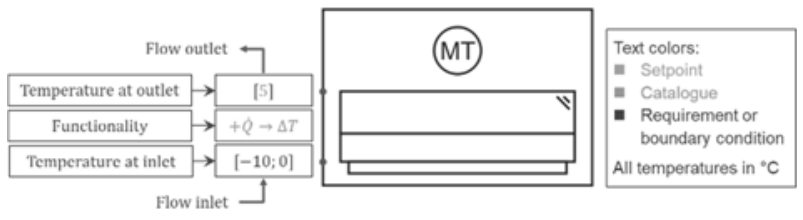

Figure 3: Simplified view of combined meta data as information basis for system synthesis.

The algorithm starts at one port and runs through all other ports of the available components in order to create valid hydraulic loops. When all options are proofed for this starting point, the algorithm restarts from another port until all possible loops are found. Note, that every component of the catalogue can be used only once in the system.

A set of rules reduce complexity and defines valid interconnections. Most important rules are described as follows:

- No interconnections between ports of the same component are allowed

- Only closed loops are allowed: The search of valid ports has to end at the same component (e.g. from evaporator outlet to inlet)

- Every loop must contain complementary functionalities (e.g. at least one positive and one negative heat flow)

- Calculated inlet temperatures have to comply to the valid range of the component port

- A port is only once connected in a loop

- No waste of generated refrigeration (e.g. evaporator to warmer outdoor unit)

- A component is allowed to have interconnections to only one of the outdoor units (best match of temperature range is chosen)

Figure 4 shows the synthesis of one loop (see button 2) with the starting point at the outlet of the MT cabinet.
This loop is valid for ambient temperatures between $0{ }^{\circ} \mathrm{C}$ and $20{ }^{\circ} \mathrm{C}$. The direct return flow from the condenser of the LT refrigeration cycle to the MT cabinet is not a valid loop (see button 1 and dashed line). In this case, the temperature range is violated and the functionalities are not complementary. In the end, the whole system layout is built by overlaying all found valid loops. Every interconnection exists only once in the system.

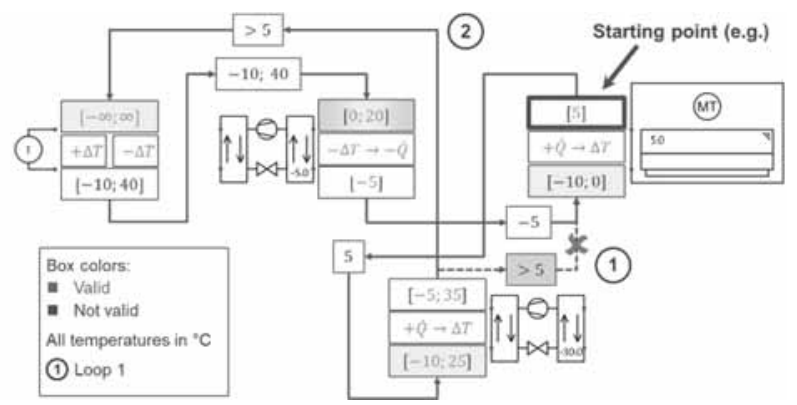

Figure 4: Example for system synthesis algorithm. Starting at the outlet of the MT cabinet, a valid flow connection to the condenser of the LT refrigeration cycle is found. From there other valid connections are found until the loop is closed. Estimated temperatures of the stream are displayed along the blue lines.

\subsection{Integration of hydraulic components}

The hydraulic concept is to use pumps for every possible interconnection and control their indicated mass flow rate with the optimizer. Thus, no valves, no controllers and no junctions are needed in the hydraulic network. From port to port, pumps are added until every mass flow rate in the hydraulic system is determined. The complete synthesized system layout is illustrated in Appendix A.

\section{Optimization of the Synthesized System}

During the applied system synthesis, no simulation is used. Therefore, the synthesized system model still has to be evaluated and maybe even further improved by results of simulation. To be able to evaluate the system layout correctly, it must operate in an optimal way while facing the defined boundary conditions. Optimal operating is defined to have minimum power consumption while observing the requirements. For finding valid starting conditions a parameter study for the control inputs of the pumps was applied. Integrations were executed in Dymola with DASSL as solver. 


\subsection{Optimization problem and algorithm}

The applied optimizer is based on the Globalized Bounded Nelder-Mead algorithm (GBNM) [6]. It is capable of constraint handling via penalty function and probabilistic restarts for a globalized optimization. Using a global optimization is crucial for the represented optimization task. Finding good starting conditions can be very time extensive and many local minima are expected for this type of systems.

Another big advantage is, even of the basic NelderMead-Algorithm [7], that it allows a non-gradient evaluation of the model. The used models, especially for the refrigeration cylces, show high non-linear behaviour. Thus, sensible gradient evaluation might restrain the optimization progress or even fail at some operating points. Two other aspects appeared to be important as well: Firstly, with gradient evaluation, a precise normalization of the cost function has a strong impact on the optimization progress. A good normalization equals a good estimation of the cost function value at optimum. This is problematic in the context of topology optimization where a focus on widely automated procedures is crucial and starting conditions can be far away from the optimum. Secondly, unphysical parameter value combinations cannot be avoided. They lead to simulation failures, which impede cost function evaluation. Instead, an artificial penalty cost function value for failed simulations has to be used which can corrupt the gradient sensitivity. Comparison studies with the SLSQP from SciPy [10] based on [5] confirmed that issues.

Since the system model does not contain energy storage components, it is assumed that there is no time constant that is relevant for the power consumption. Thus, a dynamic optimal control problem can be avoided. Instead, a parameter optimization at steady state is applied with a batch of discrete ambient temperatures.

Cost function and constraints. The main object of the optimization is to minimize the sum of all electrical power consumption in the system. Additionally, deviations to setpoint temperatures $\left(T_{\text {set }}-T\right)$ are added to the cost function via penalty function. Furthermore, mass flow rates that are not directly driven by a specific pump shall be only positive for better understanding. Those dependent mass flow rates $\left(\dot{m}_{\text {dep }}\right)$ are considered as constraints, too. Figure 5 shows how the system model evaluation during optimization is done.

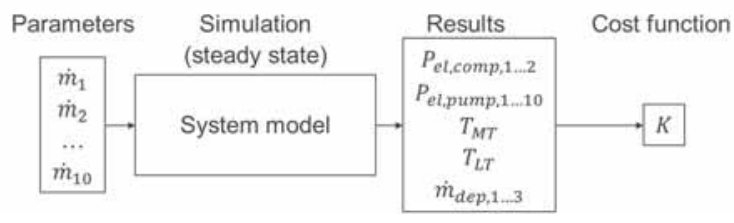

Figure 5: System evaluation during optimization. Optimization parameters are the mass flow rate inputs $(\overrightarrow{\dot{m}})$ for all pumps in the hydraulic system. Calculated variables for electrical power consumption $\left(P_{e l}\right)$, temperatures $(T)$ and dependent mass flow rates $\left(\dot{m}_{d e p}\right)$ are part of the cost function.

Weighting and normalization are part of the penalty function. The optimization problem and the cost function is defined as follows:

Minimize $K(\overrightarrow{\dot{m}})$, where

$$
\begin{aligned}
K(\overrightarrow{\dot{m}})= & \sum_{n=1}^{10} P_{e l, p u m p, n}+\sum_{n=1}^{2} P_{e l, \text { comp }, n}+ \\
& +f_{c}\left(T_{M T, \text { set }}-T_{M T}\right)+ \\
& +f_{c}\left(T_{L T, \text { set }}-T_{L T}\right)+ \\
& +\sum_{n=1}^{3} f_{c}\left(\dot{m}_{\text {dep }, n}\right)
\end{aligned}
$$

With

$$
\begin{array}{ll}
\overrightarrow{\dot{m}} & - \text { Vector with } \dot{m}_{1}, \ldots, \dot{m}_{10}[\mathrm{~kg} / \mathrm{s}] \\
P_{e l} & - \text { Electrical Power }[\mathrm{W}] \\
f_{c} & - \text { Normalized constraint penalty function }[\mathrm{W}] \\
T & - \text { Temperature }[\mathrm{K}] \\
\dot{m}_{d e p} & - \text { Dependent mass flow rate }[\mathrm{kg} / \mathrm{s}]
\end{array}
$$

\subsection{Optimization results}

All parallel global optimizations were limited to a maximum duration of about 40 hours, while 2000 iterations for one local search was set as maximum. During one global optimization (at constant ambient temperature) up to 30 local searches took place. In all global runs, several local searches ended up in the same optimum. Furthermore, no better set of parameters could be found by parameter studies. Thus, the optimization results seem to provide valid global minima. Integrations were numerically robust and fast: Failures only occurred due to unphysical parameter values. Speed of integration for one evaluation was about 1400 times real time.

Note that the presented results in this paper are manually simplified for better understanding: Very small mass flow rates are set to zero and some results are adjusted to show the corresponding operating mode clearer.

Figure 6 shows that under the chosen algorithm options, the optimizer is capable to change operating mode even under one local search. Under less precise trunca- 
tion criteria, this local search would have stopped at about 800 evaluations and a new local run would have been initiated. With the used truncation criteria, the optimizer was capable to find a new and better minimum within the same local run. Comparing the parameter values at 800 with those at the end of the run, the operating mode changed: In the beginning, the MT cabinet and the condenser of the LT refrigeration cycle is cooled in parallel from the evaporator of the MT refrigeration cycle. At the end of the run, the working fluid that flows to the LT refrigeration cycle is completely passed on to the MT cabinet. Only less than 5 $\mathrm{kW}$ of the $45 \mathrm{~kW}$ cooling load at the MT cabinet is served directly from the MT refrigeration cycle. In Figure 7 , the system simulation with optimized operating control can be seen.

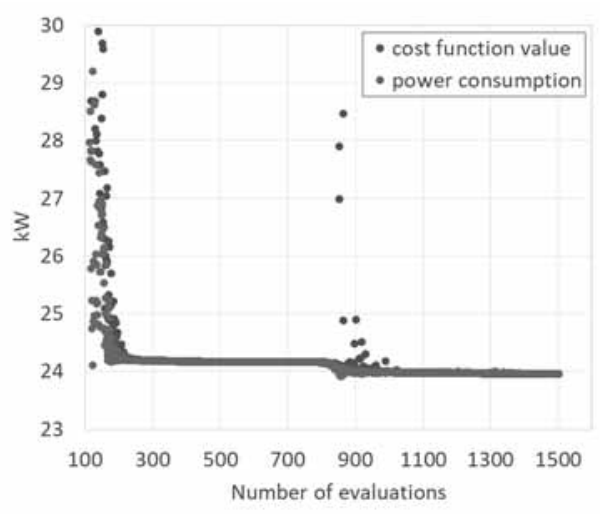

Figure 6: Detail of one local search at $20^{\circ} \mathrm{C}$ ambient temperature. After elimination of the constraint penalties, the cost function value equals the power consumption. After 800 evaluations, a set of parameters is found that represents a better operating strategy.

This operating mode is found as global optimum for all ambient temperatures above $5{ }^{\circ} \mathrm{C}$, but of course with slightly different mass flow rates.

Below $0{ }^{\circ} \mathrm{C}$ ambient temperature, the optimal operating mode is characterized by cooling the condenser of the LT refrigeration cycle and the MT cabinet by the outdoor unit. About $55 \%$ of the $45 \mathrm{~kW}$ cooling load at the MT cabinet is served by mass flow rate passed on from the condenser of the LT refrigeration cycle. Figure 8 shows the optimal mass flow rates at $-5{ }^{\circ} \mathrm{C}$ ambient temperature. At lower ambient temperatures (about $-7{ }^{\circ} \mathrm{C}$ ), even $100 \%$ feedthrough is preferred: All cooling load at the MT cabinet is driven by the mass flow rate through the LT refrigeration cycle's condenser and no direct cooling from the outdoor unit to the MT cabinet is used.

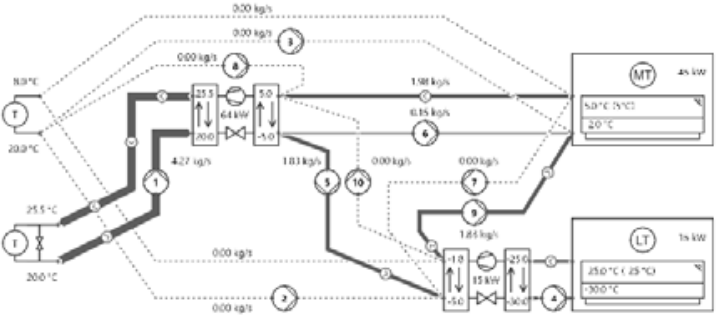

Figure 7: Optimized mass flow rates at $20^{\circ} \mathrm{C}$ ambient temperature. The mass flow rate that flows from the evaporator of the MT refrigeration cycle to the condenser of the LT refrigeration cycle is completely passed on to the MT cabinet without direct return.

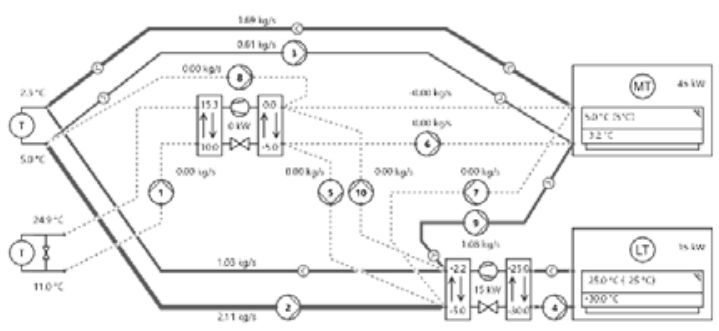

Figure 8: Optimized mass flow rates at $-5{ }^{\circ} \mathrm{C}$ ambient temperature. MT refrigeration cycle is switched off. The LT refrigeration cycle and the MT cabinet are cooled via outdoor unit. A big amount of working fluid at the condenser outlet is passed on to the MT cabinet.

At ambient temperatures around $5{ }^{\circ} \mathrm{C}$ the outdoor unit covers the needed cooling at the condenser of the LT refrigeration cycle with about $60 \%$. The rest of about 9 $\mathrm{kW}$ is served by mass flow rate from the MT cabinet outlet. This additional mass flow rate through the condenser is passed on to the outdoor unit with a few degrees above ambient temperature, cooled down and from there passed on to the inlet of the MT refrigeration cycle. From there it flows again to the MT cabinet. There is a small range of ambient temperature in which this operating mode makes sense. Additionally, the benefit for energy consumption is very small compared to the second best result (see Figure 9 in Chapter 6).

\section{Derivation of System Layout Reduction}

In this section the capability of the presented procedure to allow topology improvements is proofed. A system topology optimization typically includes a variation of number and type of components as well as their interconnections. The main purpose of this paper is not to optimize the system topology. 
However, it is a preliminary study to approach topology optimization in the future. The main idea is to eliminate components and interconnections by analysing the optimized mass flow rates.

Interconnections that show zero mass flow rates at all boundary conditions could be easily eliminated. Since the optimization contains the power consumption of the pumps, in some cases the results of the present study show a splitting of mass flow rate over several interconnections in order to avoid a very high mass flow rate at only one of the interconnections. For instance, providing cooling for the MT cabinet at low ambient temperatures is not only applied by direct cooling from the outdoor unit. Instead, additional mass flows from the outdoor unit to the MT cabinet through the evaporator of the switched off MT refrigeration cycle. In these cases, it is difficult to evaluate the necessity of particular interconnections. Therefore, the known loops from the system synthesis are used to detect corresponding operating modes and hence allow the derivation of second best operating strategies that are referenced to the optimal solution.

Three variations of the synthesized system layout are analysed. Each system layout is quantified by the sum of the power consumptions weighted according to the incidence of relevant ambient temperatures over one year for Berlin, Munich, Barcelona, Madrid and Oslo. Since no component of the simplified secondary-loop refrigeration system could be eliminated, an economical evaluation is not required.

In the first place, the optimization results show never a direct return from LT refrigeration cycle (condenser) to MT refrigeration cycle (evaporator). Instead, all mass is returned via MT cabinet. Thus, this interconnection could be simply eliminated (see Figure 9, pump 10). Secondly, looping mass from the MT cabinet back to MT refrigeration cycle (evaporator) via the condenser of the LT refrigeration cycle and outdoor unit is only used around $5{ }^{\circ} \mathrm{C}$ ambient temperature. Second-best option is a higher mass flow rate from the outdoor unit to the condenser of the LT refrigeration cycle. Figure 9 shows the original optimization results at $5{ }^{\circ} \mathrm{C}$. The elimination of the red interconnections only leads to a higher electrical energy consumption over year of about $0.1 \%$ for all considered cities.

The third option that has been analysed is the elimination of all two connections between the LT refrigeration cycle and the MT cabinet. This affects the operating mode for ambient temperatures below $0{ }^{\circ} \mathrm{C}$ and above
$10{ }^{\circ} \mathrm{C}$. In Appendix B, the corresponding layout reduction can be seen. Compared to the optimization results, the estimated energy consumption over year is slightly higher: Between 1.3\% for Oslo and $1.7 \%$ for Barcelona.

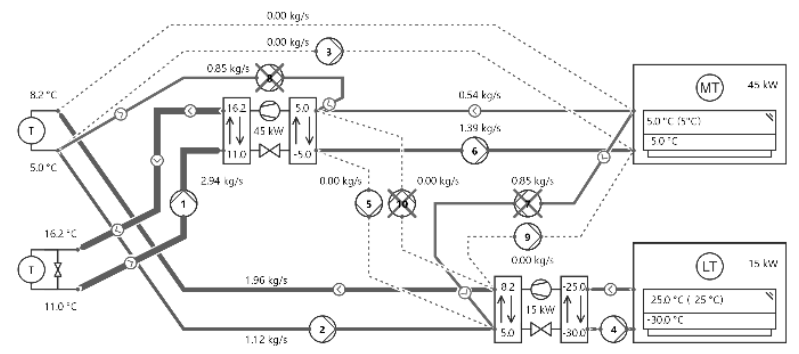

Figure 9: Optimized mass flow rates at $5{ }^{\circ} \mathrm{C}$ ambient temperature. The LT refrigeration cycle could be cooled only via outdoor unit. Thus, the red interconnections could be eliminated with almost the same electrical energy consumption. The path with pump 10 could be eliminated anyway. The reduced system layout is valid for all considered ambient temperatures.

\section{Conclusion}

The represented approach for system synthesis and optimization reduced the number of interconnections from potentially 64 to 10 . The synthesized secondaryloop refrigeration system contains all physical reasonable interconnections. The system model covers all relevant non-linearities. At the same time, integration was fast and numerically robust. The applied GBNM algorithm appeared to be robust in finding global minima within an acceptable duration of 1 day.

The optimization results allowed the derivation of further system layout reductions. The catalogued thermal functionalities of the components and the found hydraulic loops during system synthesis were important to interpret optimization results and to detect corresponding operating strategies.

\section{Acknowledgements}

This work has been supported by the German Ministry BMBF in the VEOTOP project (FKZ: 01LY1809A). We are much obliged to the $\mathrm{BMBF}$ and the project leader DLR.
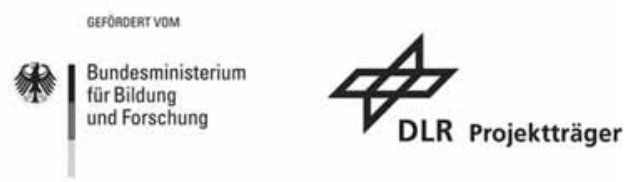

SNE 29(2) - 6/2019 99 


\section{References}

[1] Fidorra N, Kistner J, Tegethoff W, Köhler J. Energetische Bewertung von Wasserkreislauf-Systemen für $\mathrm{Su}-$ permärkte. In DKV-Tagungsberichte. Deutsche Kälteund Klimatagung; 2017; Bremen.

[2] Fidorra N, Köhler J. Energetische Untersuchung integrierter Supermarktkonzepte. In DKV-Tagungsberichte. Deutsche Kälte- und Klimatagung; 2016; Kassel.

[3] Kaiser C, Schröder A, Raabe G. Entwicklung eines CO2Ejektorkreislaufs für eine umschaltbare WärmepumpenKlimaanlage für Omnibusklimaanlagen. Deutsche Bundesstiftung Umwelt - Forschungsbericht (AZ: 30270). 2015.

[4] Karampour M, Sawalha S. Comparison of State-of-theart $\mathrm{CO} 2$ and Alternative Refrigeration Systems for Supermarkets. Gustav Lorentzen Conference; 2018; Valencia.

[5] Kraft, D. A software package for sequential quadratic programming. Forschungsbericht DFVLR. 1988; DFVLR-FB 88-28.

[6] Luersen MA, Le Riche R, Guyon F. A constrained, globalized, and bounded Nelder-Mead method for engineering optimization. Struct Multidisc Optim. SpringerVerlag; 2003.

[7] Nelder JA, Mead R. A simplex for function minimization; The Computer Journal. 1965, Volume 7 (4): 308 313

[8] Nöding M, Fidorra N, Gräber M, Köhler J. Operation Strategy for Heat Recovery of Transcritical CO2 Refrigeration Systems with Heat Storages. 29th International Conference on ECOS; 2016; Portoroz.

[9] Peteranderl C, Bernath M, Tegethoff W, Köhler J. City Bus with Modular R744 HVAC System Based on Passenger Car Components, Thermal Management Systems Symposium; 2018; San Diego.

[10] SciPy, https://docs.scipy.org/doc/scipy/reference/tutorial/optimi ze.html (visited 19.12.2018)

[11] TLK Thermo GmbH, TIL-Suite, www.tlk-thermo.de http://www.tlk-

thermo.com/index.php/de/softwareprodukte/til-suite (visited 19.12.2018)

[12] Titze M. Dynamische Simulationen zur Wärmerückgewinnung im Supermarkt. Supermarkt-Symposium ZVKKW; 2014; Darmstadt.

[13] Titze M. Energetic Analysis and Optimisation Strategies of a modern north European Supermarket [dissertation]. TU Braunschweig; 2017.

[14] Weustenfeld T. Heiz- und Kühlkonzept für ein batterieelektrisches Fahrzeug basierend auf Sekundärkeisläufen [dissertation]. Fakultät für Maschinenbau. TU Braunschweig; 2017.

\section{Appendix}

Appendix A. The figure below shows the synthesized system model. The red interconnections have no pumps. Their mass flow rate are determined by the other mass flow rates in the hydraulic system. Temperature setpoints and refrigeration capacities are displayed as well.

The LT cabinet can only be cooled by the LT refrigeration cycle. The condenser of the MT refrigeration cycle is only be cooled by outdoor unit 2 which can hold a minimum required temperature for condensation. The MT cabinet can be cooled by MT refrigeration cycle and outdoor unit 1 . The LT refrigeration cycle (condenser) can be cooled by outdoor unit 1 and MT refrigeration cycle (evaporator). Additionally, several interconnections exist for looping mass between those components at MT temperature level.

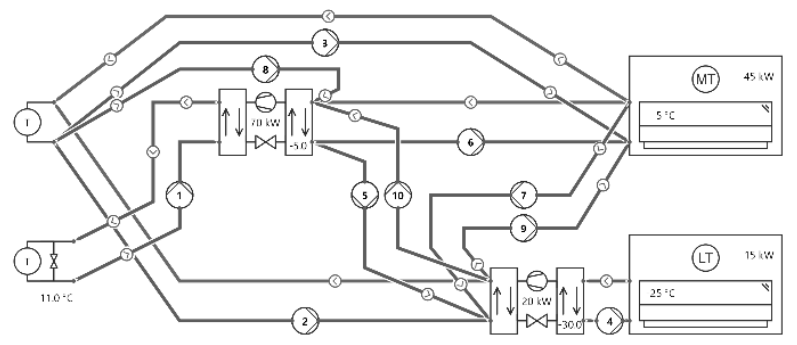

Appendix B. The figure below shows the system model of the third identified option for system reduction. Interconnections between the LT refrigeration cycle and the MT cabinet are removed. Additionally, one related interconnection is removed as well. Remaining second-best operating modes are only simple feed and return flows between the components of MT level. Therefore, the path of pump 10 has to remain in the system layout.

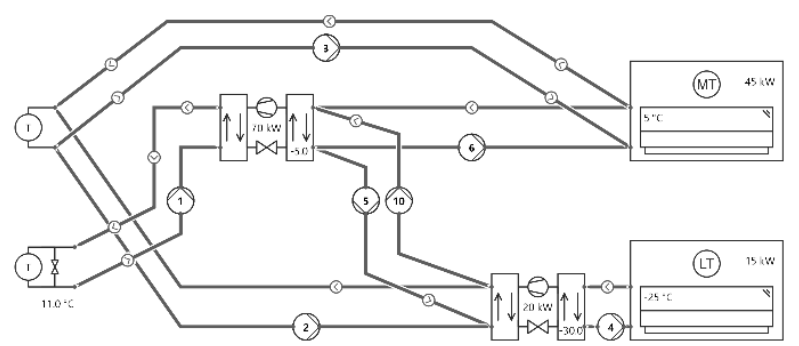

